\title{
Société de médecine et de chirurgie de Bordeaux
}

\section{Seance du 7 avril 1899.}

Syphilis oculaire grave. Paralysie de la troisième paire et këratite interstitielle.

From age t stellt eine Kranke vor, die im Verlaufe von Syphilis, nachdem eine Iritis speciñca bereits geheilt worden war, von einer completen Oculomotoriuslähmung des linken Auges und gleichzeitig von Kerat. interstit. befallen wurde. Beide Affektionen gingen unter inten-siver specifischerBehandlung und Galvanisation in vollständigeHeilung über.

Seance du 21. avril 1899.

Un nouveau cas de délire après 1'extraction de la cataracte. Fromaget berichtet über einen 77jährigen Mann der nach der Cataract-Operation in den beiden folgenden Nächten Anfälle von Delirium zeigte. In diesen beiden Tagen wurden nur 2 50-300 cbcm Urin abgesondert und Stuhlgang war gar nicht vorhanden. Das Delirium wurde als eine Intoxication von seiten der Nieren und des Darmkanals aufgefasst und verschwand unter Verabfolgung eines Purgatifs und Milchdiät.

Seance du 2 6. avril 1899.

Larmoiement hystérique. Fromaget stellte ein 26jähriges junges Mädchen vor, die am 20. Januar nach einer schweren Gemütserregung von einer Augenaffektion befallen wurde, die das Bild einer gewöhnlichen catarrhal. Conj. darbot und dementsprechend behandelt wurde. Aber trotz der Anwendung aller möaiichen Adstringentien etc. wurde das Leiden immer schlimmer; Tagcsnaehriehten.

800

der Catarrh verschwand zwar, aber die Augen blieben rot, thränten und machten die Arbeit ưoniöglicli. So blieb der Zustand der Kranken bis zum März. Zu dieser Zeit beganneơ ganz eigentümliche Erscheinungen derart, dass Anfälle von profusem Thränen unter Rotwerden und Schrnerzen der Augen auftraten, die sich alle 10-15 Minvten wieder-holten und nachts ganz wegblieben.

Diese sonderbaren vasornotorischen und secretorischen Phaenomene, die weder durch Cocain noch kalte oder warme Umsehläge gebessert • wurden, werden vom Verf. auf Grund einer genauen körperliehen Untersuchung (Anaesthesie, llyperaesthesie, concentrische Einengung des Gesíchtsfeldes, Areílexie des Rachens) auf hysterisehe Basis zurüek-gefiihrt.

Un cas de tolerance excessive de Poeïl pour un corps étranger enkysté dans le corps ciliaire pendant 16 ans.

Armaignac teilt eine Beobachtung mit, wo ein Eisensplitter, der yor 1 ti Jahren ins Auge geflogen $\Lambda$ var ( $4 \mathrm{~mm}$ lang, " $2 \mathrm{~mm}$ breit), sich im Ciliarkörper inkrustiert hatte. Nachdem in diesen 1 (i Jahren das Auge ganz reiz- und schmerzlos gewesen war, traten infolge eines neuen Traumas so heftige Schmerzanfälle ein, dass die Enucleation nötig wrurde.

Soeiété de Médecine de Marseille.

Seance du 5 mai 1899.

Rétinite pigmentaire acquise (type congenital d'origine) syphilitique. 
Mazet salt bei einem 35jähr. Patienten, der 4 Jahre vorherSyphilis acquiriert hatte, typische Retinitis pigmentosa auf beidenAugen mit alien klassischen Symj > tomen auftreten. Er meint, dassauch fur die congenitale Retin. pigment, die hereditäre Syphilis inBetracht gezogen werden müsse, wie schon von Hutchinson. Maes, Leber betont $\Lambda$ vurde. Silex.

TagesnachricW en.

Geheimrat Prof. Dr. von Rothmund - München feeging am 2G. Juli d. J. sein vierzigjähriges Jubiläum als Professor der Augen-heilkunde.

Dr. J. RoInner wurde zum Director der ophthalmologischen Klinik in Xancy ernannt.

D. Der klinische Professor des Ujasdow-Militairhospitals in Warschau

Dr. 11. Kostenicz

ist zum ausserordentlichen Professor der Augen-heilkunde ernannt worden. 